\title{
Biophysical characterization of the interaction between Forkhead Box 04 (FOXO4) and p53 transcription factors
}

\author{
Raju Mandal ${ }^{1}$, Klara Kohoutova ${ }^{1,2}$, Olivia Petrvalska ${ }^{1,2}$, Matej Horvath ${ }^{1}$, Václav Veverka ${ }^{3,4}$, Veronika Obsilova $^{2}$, and \\ Tomas Obsil ${ }^{1,2}$
}

\author{
${ }^{I}$ Department of Physical and Macromolecular Chemistry, Faculty of Science, Charles University, Prague, Czech Republic \\ ${ }^{2}$ Department of Structural Biology of Signaling Proteins, Division BIOCEV, Institute of Physiology of the Czech Academy of Sciences, \\ 25250 Vestec, Czech Republic \\ ${ }^{3}$ Institute of Organic Chemistry and Biochemistry of the Czech Academy of Sciences, Prague, Czech Republic \\ ${ }^{4}$ Department of Cell Biology, Faculty of Science, Charles University, Prague, Czech Republic \\ raju.mandal@natur.cuni.cz
}

The transcription factor p53 controls numerous cellular processes including apoptosis, senescence, DNA repair, and tumor suppression [1]. The function of p53 is closely intertwined with Forkhead box O (FOXO) transcription factors and FOXO protein regulates cellular functions such as cellular homeostasis, oxidative stress resistance, metabolism, and longevity. FOXO protein family has four members: FOXO1, FOXO3, FOXO4, and FOXO6 wherein their activity is tightly regulated by post-translation modification (phosphorylation, acetylation, methylation, and ubiquitination) [2]. The previous study has demonstrated that the physical interaction between FOXO4 and p53 represses apoptosis of senescent cells by upregulating the transcription of p21 gene and maintain the viability of senescent cells [3]. However, the structural aspects of FOXO4:p53 complex formation remain unclear. Therefore, we designed several truncated constructs of FOXO4 and p53 to elucidate the structural details of this complex using analytical ultracentrifugation, NMR, chemical cross-linking, and molecular docking. Our data suggest that the transactivation domain (TAD) of p53 and DNA binding domain of FOXO4 provide overall stability of the complex along with the transient interaction from other regions of FOXO4 and p53. Furthermore, FOXO4:p53 complex formation does not affect the DNA binding affinity of FOXO4, thereby this interaction presumably allows co-localization of both FOXO4 and p53 transcription factors in the promoter region. Our finding further promotes future research for drug development aiming for the selective elimination of senescent cells.

[1] Boutelle, A.M., Attardi, L.D. (2021) Rev. Trends Cell Biology. 31, 298-310.

[2] Obsil T., Obsilova V. (2011) Rev. Biochimica et Biophysical Acta (BBA)-Molecular cell research. 1813, 1946-1953.

[3] Baar M.P., Brandt R.M.C., Putavet D.A., et al. (2017) Cell. 169, 132-147.

Keywords: Protein-protein interaction, Forkhead Box O (FOXO) and p53 transcription factors, Analytical ultracentrifugation, NMR, Chemical cross-linking

This study was supported by Czech Science Foundation Grant No. 21-02080S and the Grant agency of the Charles University (project number: 1002119). 